\title{
Multi-axial hybrid fire testing based on dynamic relaxation
}

\section{Journal Article}

Author(s):

Tsokanas, Nikolaos (D); Abbiati, Giuseppe (D); Kanellopoulos, Constantinos (D); Stojadinovic, Bozidar (i)

Publication date:

2021-12

Permanent link:

https://doi.org/10.3929/ethz-b-000510230

\section{Rights / license:}

Creative Commons Attribution 4.0 International

Originally published in:

Fire Safety Journal 126, https://doi.org/10.1016/j.firesaf.2021.103468

\section{Funding acknowledgement:}

764547 - Dynamic virtualisation: modelling performance of engineering structures (EC)

813424 - INNOVATIVE GROUND INTERFACE CONCEPTS FOR STRUCTURE PROTECTION (EC) 
Technical Note

\title{
Multi-axial hybrid fire testing based on dynamic relaxation
}

\author{
N. Tsokanas ${ }^{\text {a }}$, G. Abbiati ${ }^{\text {b, }}$, K. Kanellopoulos ${ }^{\text {a }}$, B. Stojadinović ${ }^{a}$ \\ ${ }^{a}$ ETH Zurich, Institute of Structural Engineering, Chair of Structural Dynamics and Earthquake Engineering, Stefano-Franscini-Platz, 5, 8093, Zurich,Switzerland \\ ${ }^{\mathrm{b}}$ University of Aarhus, Department of Civil and Architectural Engineering, Inge Lehmanns Gade, 10, 8000, Aarhus C, Denmark
}

\section{A R T I C L E I N F O}

\section{Keywords:}

Hybrid fire testing

Dynamic relaxation

Partitioned time integration

\begin{abstract}
A B S T R A C T
This technical note presents the experimental validation of a hybrid fire testing coordination algorithm recently developed by some of the authors. For the first time, the algorithm is applied to solve the static response of a multiple-degrees-of-freedom hybrid model.
\end{abstract}

\section{Introduction}

Hybrid fire testing (HFT) replaces a prototype structure under test with a hybrid model that combines physical and numerical substructures (PS and NS, respectively). The PS is tested in the laboratory using servocontrolled actuators and furnaces. The NS is instantiated in a structural analysis software. A coordination algorithm ensures kinematic compatibility and force balance between PS and NS. There is a general consensus among researchers that coordination algorithms should rely on a constant PS tangent stiffness matrix estimate, possibly equal to its initial value, and facilitate the reuse of existing structural analysis software for simulating the NS. Consistent with this philosophy, a HFT coordination algorithm was recently proposed by Abbiati et al. [1], which relies on dynamic relaxation and partitioned time integration. The experimental validation of the algorithm on a single-degreeof-freedom (single-DoF) hybrid model was recently carried out at the
Danish Institute of Fire Safety and Security Technology by Sauca et al. [2].

This technical note reports the results of the experimental validation of the same HFT algorithm on a multiple-DoFs hybrid model. For a comprehensive state-of-art review on HFT, the reader shall consult Refs. $[1,2]$.

\section{Coordination algorithm}

The HFT algorithm proposed in Ref. [1] combines the dynamic relaxation method and partitioned time integration to solve the static coupled equation of motion of the hybrid model. The basic idea behind dynamic relaxation is to obtain the displacement solution of a static structural problem by computing the transient response of an equivalent dynamic system. For a hybrid model comprising a single PS and a single NS, the system of coupled equations of motion reads,

$$
\left\{\left\{\mathbf{M}^{N} \ddot{\mathbf{u}}^{N}+\mathbf{C}^{N} \dot{\mathbf{u}}^{N}+\mathbf{r}^{N}\left(\mathbf{u}^{N}\right)=\mathbf{f}^{N}+\mathbf{L}^{N^{T}} \boldsymbol{\Lambda}^{N} \quad \mathbf{L}^{N} \dot{\mathbf{u}}^{N}+\overline{\mathbf{L}}^{N} \dot{\mathbf{u}}^{g}=\mathbf{0} \quad\left\{\mathbf{M}^{P} \ddot{\mathbf{u}}^{P}+\mathbf{C}^{P} \dot{\mathbf{u}}^{P}+\mathbf{r}^{P}\left(\mathbf{u}^{P}\right)=\mathbf{f}^{P}+\mathbf{L}^{P^{T}} \boldsymbol{\Lambda}^{P} \quad \mathbf{L}^{P} \dot{\mathbf{u}}^{P}+\overline{\mathbf{L}}^{P} \dot{\mathbf{u}}^{g}=\mathbf{0} \quad \overline{\mathbf{L}}^{N^{T}} \boldsymbol{\Lambda}^{N}+\overline{\mathbf{L}}^{P^{T}} \boldsymbol{\Lambda}^{P}=\mathbf{0}\right.\right.\right.
$$

\footnotetext{
* Corresponding author.

E-mail address: abbiati@cae.au.dk (G. Abbiati).
} 

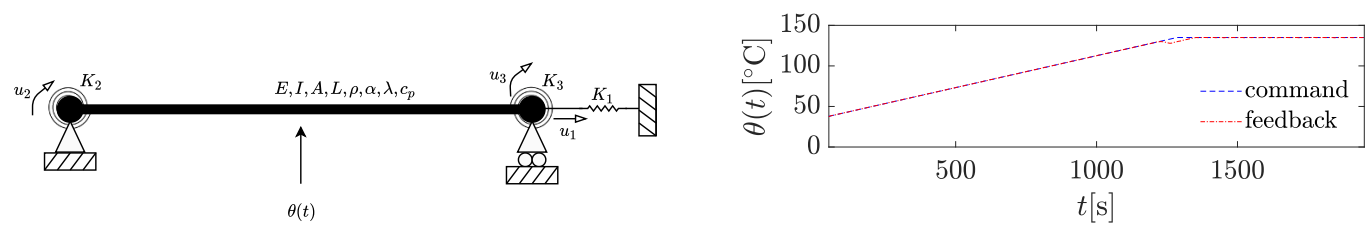

Fig. 1. Validation case study: a) prototype structure; b) temperature history.
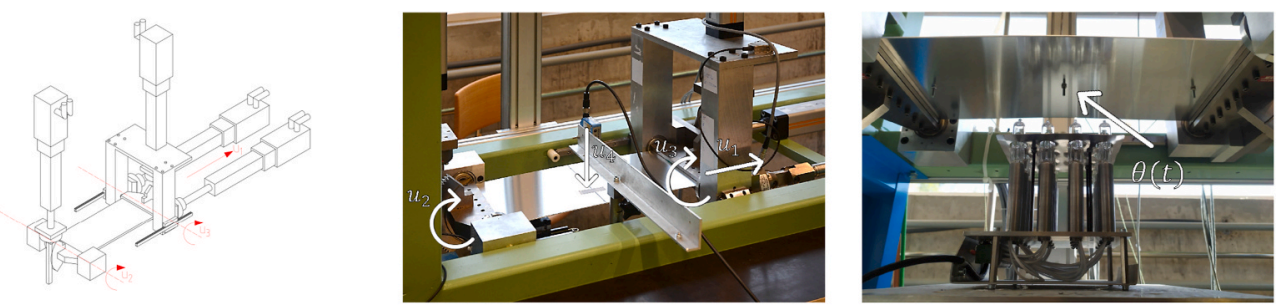

Fig. 2. Experimental setup: a) 3-DoFs test rig; b) plate specimen; c) infrared lamp.

where $\mathbf{r}$ are internal restoring force vectors, while $\mathbf{f}(t)$ represent time-varying external loading; $\mathbf{M}$ and $\mathbf{C}$ are fictitious mass and damping diagonal matrices,

$M_{i i}=\frac{(1.1 \Delta t)^{2}}{4} \sum_{j}\left|K_{i j}\right|, \quad C_{i i}=2 \omega_{0} M_{i i}$

where $K_{i j}$ is a generic entry of the initial tangent stiffness matrix $\mathbf{K}=\partial \mathbf{r}$ / $\partial \mathbf{u} ; \Delta t$ is the time step size of the equivalent transient analysis; $\omega_{0}$ is the lower eigenvalue of the coupled equations of equilibrium, as defined in (1), which is estimated with a monolithic linearization of the hybrid model. $\mathbf{L}$ and $\overline{\mathbf{L}}$ are Boolean signed matrices, which localize interface DoFs on every single substructure and vector $\mathbf{u}_{g}$, respectively. The latter gathers all DoFs that are shared between at least two substructures, namely the interface DoFs.

\section{Experimental validation}

The experimental validation of the coordination algorithm relies on the 3-DoFs prototype structure reported in Fig. 1, which consists of a simply supported beam with elastic restraints. Specifically, $u_{1}, u_{2}$ and $u_{3}$ indicate the axial and the two rotational DoFs of the hybrid model while $r_{1}, r_{2}$ and $r_{3}$, the corresponding restoring force and bending moments. The PS coincides with the beam element and consists of an aluminum plate of $b \times h=200 \times 2 \mathrm{~mm}$ rectangular cross-section and $L=460 \mathrm{~mm}$ length. The main material parameters of aluminum are Young modulus $E=69.50 \mathrm{GPa}$, density $\rho=2700 \mathrm{~kg} / \mathrm{m}^{3}$, thermal expansion $\alpha=23 \times$ $10^{-6}{ }^{\circ} \mathrm{C}^{-1}$, thermal conductivity $\lambda=201 \mathrm{~W} / \mathrm{m}^{\circ} \mathrm{C}$, specific heat $c_{p}=910$ $\mathrm{J} / \mathrm{kg}^{\circ} \mathrm{C}$. The NS coincides with the elastic restraints, that is, the lateral spring of stiffness $K_{1}=2500.00 \mathrm{kN} / \mathrm{mm}$ and both rotational spring of stiffness $K_{2}=129.43$ and $K_{3}=408.52 \mathrm{kNmm} / \mathrm{rad}$. Thermal loading is imposed to the PS by means of a $2.4 \mathrm{~kW}$ infrared lamp module. The temperature measured at the center of the plate is linearly ramped from room temperature $\theta_{0} \approx 20{ }^{\circ} \mathrm{C}$ to $\theta_{\max }=135{ }^{\circ} \mathrm{C}$ in about $1400 \mathrm{~s}$. The following hold phase lasts for $600 \mathrm{~s}$. Noteworthy, the stiffness of the axial spring $K_{1}$ is about 50 times larger than the axial stiffness of the plate. As a result, the latter buckles if subjected to thermal expansion. The stiffness of each rotational spring is close to the bending stiffness of the plate, so its buckling length has an intermediate value between pinpin $(L)$ and fixed-fixed $(L / 2)$ boundary conditions. A time step $\Delta t=1$ msec is used to integrate (1). Before HFT starts, the coordination algorithm solves the hybrid model response with no thermal and mechanical loading until actuator restoring forces approach zero. Fig. 2 shows the experimental setup (see Ref. [3] for more details).

\section{Results and discussion}

The effectiveness of the algorithm is demonstrated by examining response quantities related to a single HFT. In this regard, Fig. 3 compares PS and NS displacement response quantities, while Fig. 4 compares PS and NS force response quantities. For the sake of comparison, in the latter case, PS response quantities are multiplied by -1 so that matching with NS response quantities can be graphically verified. As one can note, some discontinuity characterizes both $u_{3}$ and $r_{3}$, which are associated with the right rotational DoF of the hybrid model. The reason is that the bearings of the right clevis of the 3-DoFs test rig are not perfectly aligned and the joint suffers from stick and slip behavior. However, as can be observed, both compatibility and balance between PS and NS are attained with a high degree of accuracy. Finally, for the sake of comparison, residual forces, which are computed as the sum of fictitious inertia and damping forces of both PS and NS, are reported in Fig. 5. As can be observed, residual forces are small compared to restoring forces of Fig. 4.

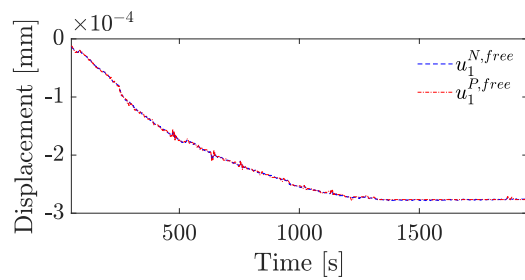

(a)

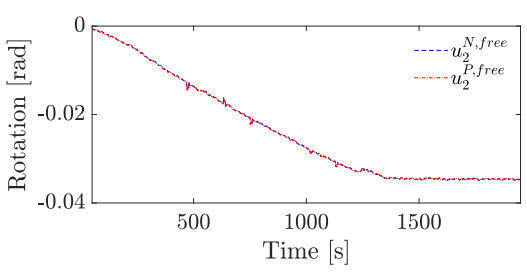

(b)

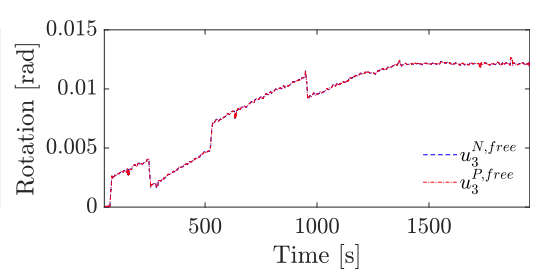

(c)

Fig. 3. Displacement response a) displacement DoF $u_{1}$; b) rotation DoF $u_{2}$; c) rotation DoF $u_{3}$. 


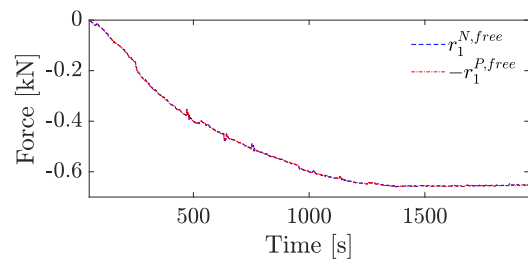

(a)

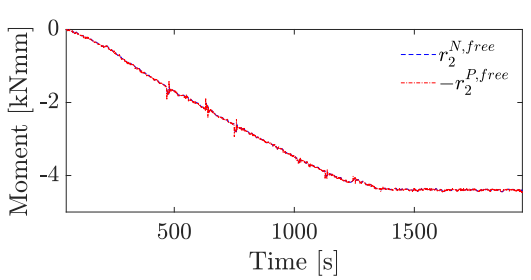

(b)

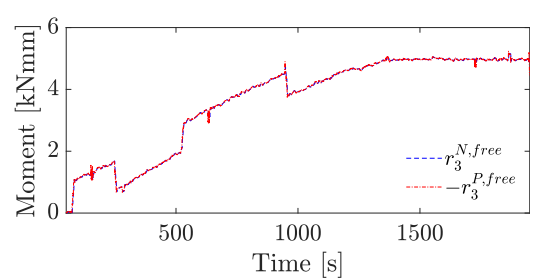

(c)

Fig. 4. Restoring forces: a) displacement DoF $u_{1}$; b) rotation DoF $u_{2}$; c) rotation DoF $u_{3}$.

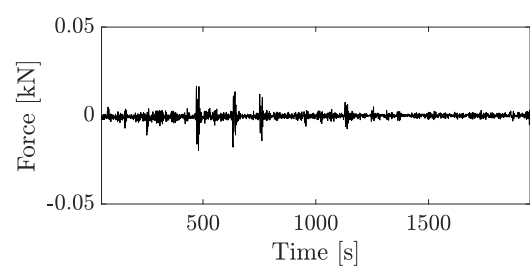

(a)

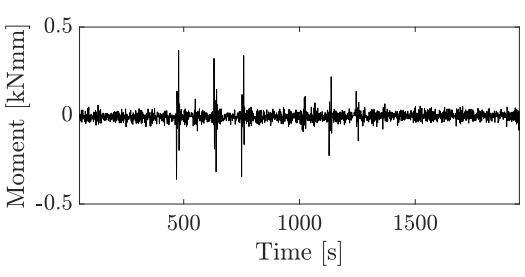

(b)

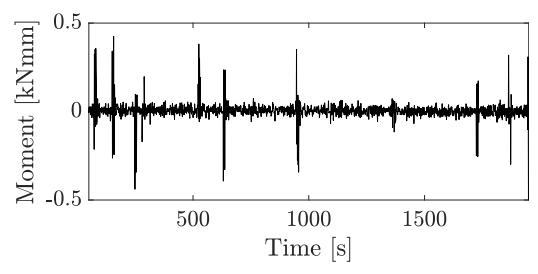

(c)

Fig. 5. Residual forces: a) displacement DoF $u_{1}$; b) rotation DoF $u_{2}$; c) rotation DoF $u_{3}$.

\section{Conclusions and outlooks}

Experimental results demonstrate that, with the proposed coordination algorithm, compatibility and balance between physical and numerical substructures are attained with a high level of accuracy. Also, residual forces are small compared to restoring forces. Hybrid fire testing with nonlinear numerical substructures will be the target of our future work.

\section{Author statement}

N. Tsokanas: Visualization, Validation, Software, Writing - Original Draft. G. Abbiati: Conceptualization, Methodology, Software, Writing Original Draft. K. Kanellopoulus: Validation, Software. B. Stojadinovic: Supervision, Project administration, Funding acquisition.

\section{Declaration of competing interest}

The authors declare that they have no known competing financial interests or personal relationships that could have appeared to influence the work reported in this paper.

\section{Acknowledgments}

The first and third authors were supported by the Marie SkłodowskaCurie grants 764547 and 813424 , respectively. The 3-DoFs test rig was funded by the Swiss Space Office, THERMICS MdP2016 Project, grant REF-1131-61001.

\section{References}

[1] G. Abbiati, P. Covi, N. Tondini, O. Bursi, B. Stojadinovic, A real-time hybrid fire simulation method based on dynamic relaxation and partitioned time integration, ASCE Journal of Engineering Mechanics.

[2] A. Sauca, N. Mortensen, A. Drustrup, G. Abbiati, Experimental validation of a hybrid fire testing framework based on dynamic relaxation, Fire Safety Journal 121 (2021) 103315.

[3] G. Abbiati, S. Marelli, N. Tsokanas, B. Sudret, B. Stojadinović, A global sensitivity analysis framework for hybrid simulation, Mech. Syst. Signal Process. 146 (2021) 106997. 